\title{
Numerical simulation of acoustic scattering and flow- induced noise with sharp interface immersed boundary method
}

\author{
Cheng Zhao a, c, 1, Yan Yang ${ }^{\mathrm{b}, \mathrm{c}, 2}$, Tao Zhang ${ }^{\mathrm{a}, 3}$, Haibo Dong ${ }^{\mathrm{c}, 4}$ \\ a School of Naval Architecture \& Ocean Engineering, Huazhong University of Science \& Technology, Wuhan, Hubei 430074, China \\ ${ }^{\mathrm{b}}$ LHD, Institute of Mechanics, Chinese Academy of Sciences, Beijing 100190, China \\ c Department of Mechanical and Aerospace Engineering, University of Virginia, Charlottesville, VA 22904, USA
}

\begin{abstract}
High efficient and quiet propulsion of flying and swimming animals promotes the investigations on acoustic scattering and flow-induced noise. One challenge for aero/hydroacoustic simulation is the treatment on complex and moving solid boundaries, with retaining high-order accuracy. In this paper, a sharp interface immersed boundary method (IBM) was implemented in both fluid dynamics and aero/hydro-acoustics, within a hybrid approach. The hybrid approach combined a high-fidelity DNS solver for flow field and a low-dispersion, lowdissipation acoustic solver, with optimized computational aeroacoustic schemes, using the acoustic perturbation equations (APEs). A series of benchmark problems on aeroacoustic propagation and scattering acoustic fields with a cylinder or sphere scatterer were computed to validate the accuracy and boundary condition treatments of the acoustic solver, in two dimension and three dimension. Following this, the flow field and flow-induced noise of flow past a stationary cylinder, with Reynolds number 200 was presented. With the hybrid approach proposed in the paper, the potential for dealing with bio-inspired problems in flying and swimming animals is indicated.
\end{abstract}

Keywords: acoustic perturbation equations, immersed boundary method, acoustic scattering, flow-induced noise

\section{Introduction}

Flying and swimming animals with propulsion of high efficiency and quietness promote the investigations on acoustic scattering and flow-induced sound. Through two decades and recently, many works have been done on the hydro/aero-dynamics inspired by fast and efficient propulsion, in flying and swimming [1, 2], specified fish fins [3, 4] and bird/insect wing [5, 6]. However, the study on aero/hydrodynamic noise has received less attention. The studies on acoustic scattering and flow-induced noise problems play an important role in underwater navigation system and ocean acoustics. Besides, the studies of flow-induced noise can be applied to the design of quiet underwater vehicles and bio-inspired robots.

The theoretical method [7,8], experimental measurement [9-11] and numerical simulation [12-20] were used to investigate the acoustic scattering and flow-induced noise in previous studies. Comparing with the former two methods, numerical simulation has its particular advantages on multiple aspects, such as parametric study and mechanism research. Currently, the numerical techniques of acoustic simulation including direct numerical simulation (DNS) $[14,17]$ and hybrid method, later is widely applied due to the high cost of DNS [21]. The hybrid method can be mainly classed into acoustic analogy method [18-20], linearized Euler Equations (LEEs) [16, 22] and hydrodynamic/acoustic splitting approach [12, 13, 23, 24]. Comparing with acoustic analogy method, the other two methods could obtain the noise source information

\footnotetext{
${ }^{1}$ Ph.D. Student, zhaoch@hust.edu.cn, AIAA student Membership

${ }^{2}$ Associate Professor, yangyan@imech.ac.cn

${ }^{3}$ Associate Professor, zhangt7666@,hust.edu.cn

${ }^{4}$ Associate Professor, haibo.dong@,virginia.edu, AIAA Associate Fellow
} 
and consider the convection and refraction effects of a non-uniform flow field, which is more suitable for mechanism analysis of sound generation and propagation.

In previous researches, Hardin and Pope [12] proposed an acoustic/viscous splitting technique for the numerical analysis of aerodynamic noise generation and validate the approach by the oscillating sphere and sound generation by flow over a two-dimensional cavity. Bailly and Juve [22] studied the acoustic propagation problems by solving LEEs with various source terms and compared with analytical solutions to check accuracy of numerical simulations. In order to suppress the instability of LEEs from entropy and vorticity waves, Ewert and Schröder [13] formulated a family of acoustic perturbation equations for the simulation of flow-induced acoustic fields and predicted the trailing edge noise [25] by this method. Seo and Mittal [26] studied the acoustic wave scattering and low-Mach number flow-induced sound by solving linearized perturbed compressible equations (LPCEs) with immersed boundary method (IBM). This method was then applied to study the effect of wing flexibility on sound generation of flapping wings [27].

From aforementioned references, researches in aeroacoustics have seen been renewed activity in recent years. However, one challenge of acoustic simulation is the boundary treatment, especially for complex and moving boundary. The unstructured grids and overset grids are two methods that can be applied to deal with this condition. Unstructured grids are difficult to extend to high-order accuracy and overset grids method usually require high order interpolation and large data exchange. An important method to deal with complex geometry and moving boundary in computational fluid dynamics (CFD) is immersed boundary method. These methods have been successfully applied to solve CFD problems with complex geometry and moving boundary.

In this work, the main work is concerned with a unique combination of APEs with immersed boundary method treatment and its numerical implementation to simulate the acoustic scattering and flow-induced noise problems. The APE system [13] is used to solve acoustic field based on flow field, with proper optimized computational aeroacoustic (CAA) schemes and techniques. The sharp interface immersed boundary method is used to in both the CFD and CAA solver. Benchmark two-dimensional and threedimensional problems in acoustic scattering are shown in order to test the present acoustic code. Following this, the flow-induced noise of flow past cylinder is computed and compared with reference solutions. An outline of the chapters is as follows. Sec. II describes the detailed computational method including governing equations, numerical methodology and boundary treatment. In Sec. III, a series of cases were shown. Firstly, the validation cases of CAA solver are presented. Secondly, the flow-induced noise of a cylinder was simulated to test the present hybrid CFD/CAA approaches. The summary is given in Sec. IV.

\section{Computational method}

\section{A. Governing equations}

In this paper, a hybrid method is applied to simulate the fluid and acoustic field, the flow field is first simulated to obtain the acoustic sources, then the acoustic field is computed by solving acoustic equations. The governing equation for fluid is incompressible Navier-Stokes equations, written in a vector form as,

$$
\begin{gathered}
\nabla \cdot \boldsymbol{U}=0 \\
\frac{\partial \boldsymbol{U}}{\partial t}+(\boldsymbol{U} \cdot \nabla) \boldsymbol{U}=-\frac{1}{\rho_{0}} \nabla P+v_{0} \nabla^{2} \boldsymbol{U}
\end{gathered}
$$

where $\rho_{0}, \boldsymbol{U}, P$ are the incompressible flow density, velocity vector and pressure. $v_{0}$ is the kinematic viscosity of fluid.

The acoustic perturbation equation (APE) system is applied to solve the acoustic variables based on incompressible flow field. It is shown [13] that the excitation of instabilities in global unstable mean flows is prevented due to the properties of the APE system. The governing equations can be written in a vector form as, 


$$
\begin{gathered}
\frac{\partial \boldsymbol{u}^{\mathrm{a}}}{\partial t}+\nabla\left(\overline{\boldsymbol{u}} \cdot \boldsymbol{u}^{\mathrm{a}}\right)+\nabla\left(\frac{p^{\prime}}{\bar{\rho}}\right)=\boldsymbol{S}_{m o m} \\
\frac{\partial p^{\prime}}{\partial t}+\bar{c}^{2} \nabla \cdot\left(\bar{\rho} \boldsymbol{u}^{\mathrm{a}}+\overline{\boldsymbol{u}} \frac{p^{\prime}}{\bar{c}^{2}}\right)=S_{\text {cont }}
\end{gathered}
$$

where $p$ is the fluid pressure, $\rho$ is the density of the fluid, $\boldsymbol{u}$ is the fluid velocity, $c$ is the speed of sound, $\boldsymbol{u}^{\mathrm{a}}$ is an irrotational acoustic perturbation. Primed quantities denote perturbation quantities, while the bar symbol denotes time-average of flow quantities. The right-hand terms $S_{\text {cont }}$ and $\boldsymbol{S}_{\text {mom }}$ are respectively acoustic sources in the continuity and momentum equation(s). The left-hand sides of APE system describe the wave propagation and refraction in a non-uniform mean flow. For the low-Mach number flow noise problems, when entropy effects are discarded and only vortex sound is considered, the major source term is $\nabla P^{\prime} / \rho_{0}$ [13], where $P^{\prime}=P-\bar{P}$ is the incompressible perturbation pressure, and $\rho_{0}$ is the constant mean flow density.

\section{B. Numerical methodology}

A second-order central difference based on sharp interface immersed boundary method solver [28] is employed to solve the incompressible Navier-Stokes equations, this method has been successfully applied in solving bio-inspired problems [3, 4] and internal flow problems [29], the detailed numerical can be found in reference [28].

For acoustic simulation, the governing equations are discretized with a high order finite-difference method. The spatial discretization is calculated with the fourth order (seven-point stencil) dispersionrelation-preserving (DRP) scheme [30], the back DRP schemes are applied near the solid boundary and domain boundary [31]. In order to filter out spurious high-frequency grid-to-grid oscillations, the standard 10th-order filters using 11-point stencils [32] is applied in the interior region and the size of the stencil is decreased to 3, 5 or 7points at the boundary. Temporal integration is carried out with the six-stage low dispersion-dissipation Runge-Kutta (LddRK6) scheme [33]. For non-reflecting boundary condition at the domain boundary, the radiation conditions proposed by Tam and Dong [34] and developed for 3D geometry by C. Bogey [35] are used.

As the flow is assumed to be inviscid for acoustic simulation, the slip boundary conditions are used on the solid wall:

$$
\frac{\partial \rho^{\prime}}{\partial n}=0, \frac{\partial p^{\prime}}{\partial n}=0, \boldsymbol{u}^{\prime} \cdot \boldsymbol{n}=0
$$

where $\boldsymbol{n}$ is the unit wall-normal vector, and the initial conditions are $\rho^{\prime}=0, \boldsymbol{u}^{\prime}=\mathbf{0}$ and $p^{\prime}=p_{0}^{\prime}$. At the solid walls, one ghost point is used to satisfy the slip conditions in Equations 5.

\section{Immersed boundary treatment}

In this paper, both flow field and acoustic field boundary use the immersed boundary treatment based on ghost cells [28]. The treatment is described as follows. Firstly, the domain cells are classified as fluid cells, solid cells and ghost cells. The solid cells are the cells inside the solid body and fluid cells are outside the body. The ghost cells are defined as cells inside the solid body but has at least one neighbor fluid cell. Then a line segment is extended from the ghost cell into the fluid cell and intersects normal to the solid boundary. The position of image point is defined that the boundary intercept is midway between the ghostcell and the image-point, as shown in Fig. 1. The variables on the image point are then computed by bilinear/trilinear interpolation from surrounding fluid nodes. The values on the ghost cell then can be easily obtained by no slip boundary conditions for flow field and slip boundary conditions for acoustic simulation. The detailed boundary treatment can be found in reference [28]. 


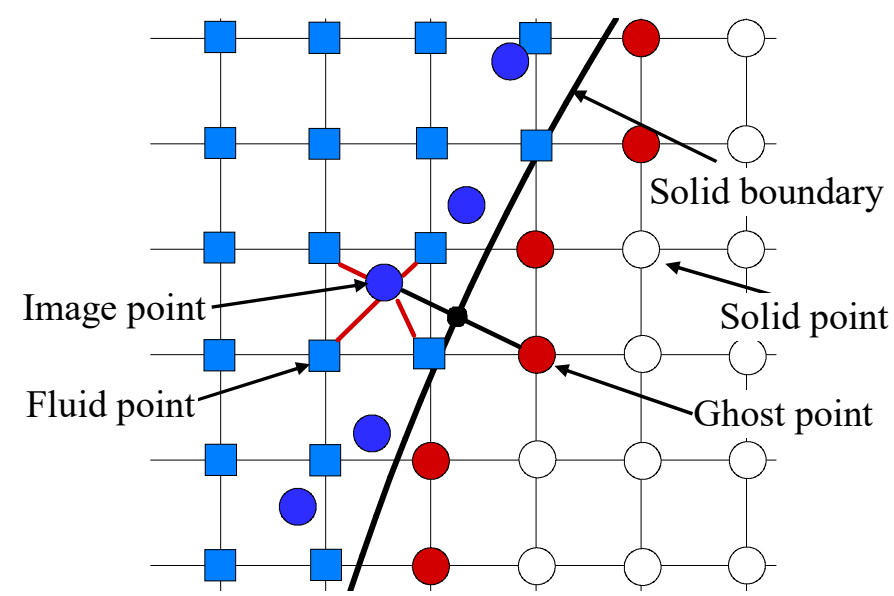

Fig.1 Schematic of the solid boundary treatment

\section{Results and Discussion}

In this section, the acoustic propagation in the free field and two benchmark problems in acoustic scattering are considered and compared with analytical solutions to test the present acoustic code and boundary treatment. A non-dimensional analysis is used to describe the unknown variables, the equations are non-dimensionalized by reference length $l$, the sound speed $c_{0}$ and the ambient density $\rho_{0}$. The time and pressure are non-dimensionalized with $l / c_{0}$ and $\rho_{0} c_{0}^{2}$.

\section{A. Acoustic propagation in the free field}

In this case, the propagation of a two-dimensional Gaussian pulse in a uniform mean flow $(M a=0.5)$ [36] is computed to test the numerical schemes and the far field non-reflecting boundary conditions. The computational domain is $-50 \leq(x, y) \leq 50$ and an artificial acoustic pulse is put in the center of the domain $x=y=0$ with initial conditions:

$$
p^{\prime}=\exp \left[-(\ln 2) \frac{x^{2}+y^{2}}{3^{2}}\right], u^{\prime}=v^{\prime}=0
$$

The APEs are solved on a $100 \times 100$ uniform mesh with $d x=d y=1.0$, and the time step is 0.5 with CFL-number is 0.75 . The instantaneous pressure contours and pressure perturbations along $y=0$ at $t=30$, 50 and 100 are presented in Fig. 2(a)-2(f). Results show that the pulse is propagating in all directions at a velocity equal to the sum of the sound speed and of the flow velocity. When the wave arrives at the right domain boundary (Fig. 2a), up and down boundary (Fig. 2b) and left boundary (Fig. 2c) the pulse leaves the computational domain without noticeable reflections. Pressure perturbations along $y=0$ at corresponding time agrees well with analytical solutions, indicating the numerical schemes and radiation boundary conditions are well realized.

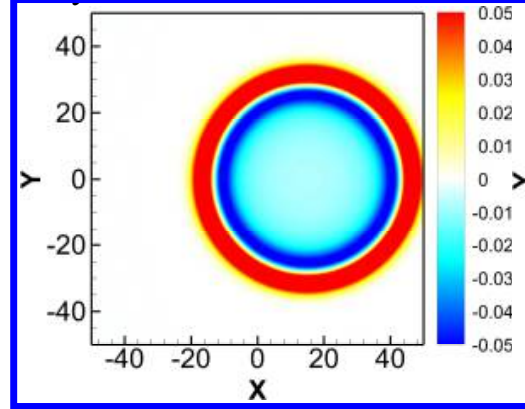

(a)

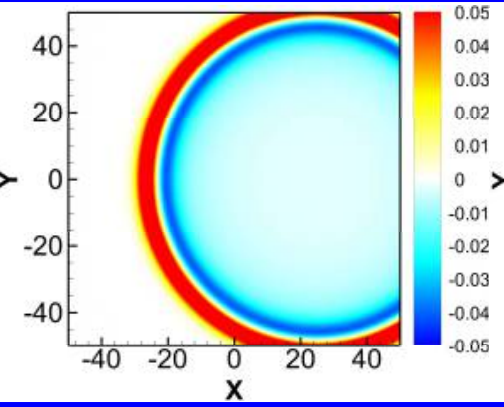

(b)

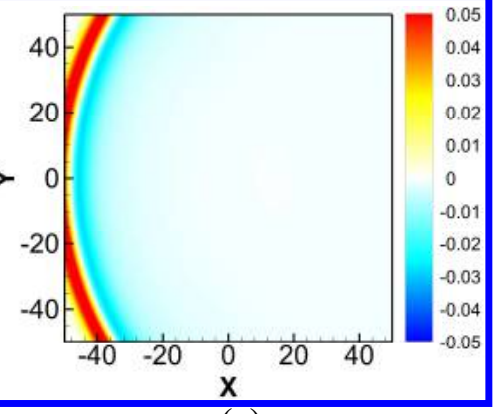

(c) 


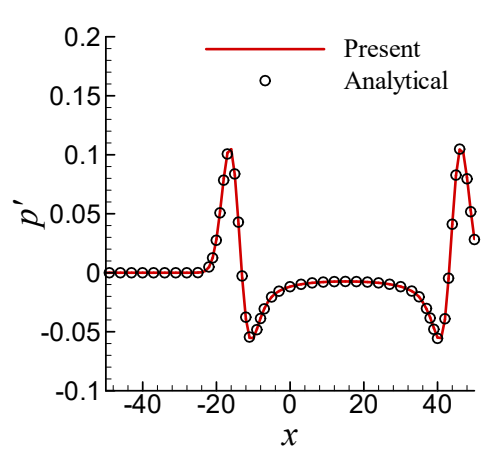

(d)

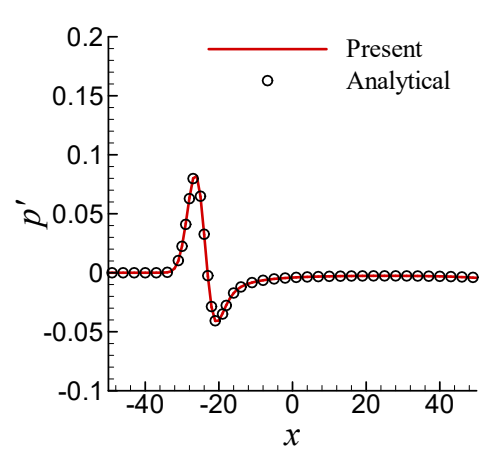

(e)

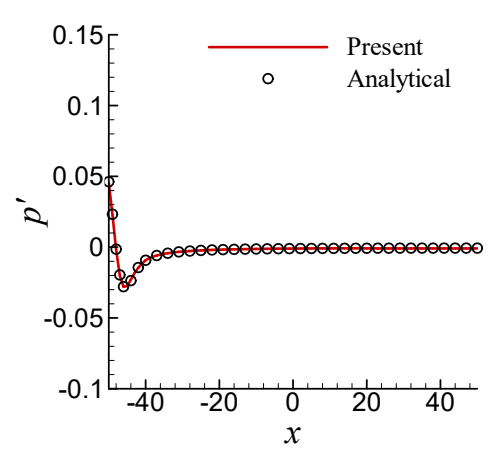

(f)

Fig. 2 Pressure perturbation contours at (a) $t=30$, (b) $t=50$, (c) $t=100$ and corresponding pressure perturbation along $y$ axis at (d) $t=30$, (e) $t=50$ (f) $t=100$

The uniform grid with $d x=d y=1.0,0.5,0.25$ and 0.125 are used to test the grid convergence. The time step is set as 0.005 to minimize the effects of time on the solution. The $L 2$ norm error is defined as:

$$
L 2=\sqrt{\frac{1}{N} \sum_{n=1}^{N}\left(p_{n}^{\text {'ana }}-p_{n}^{\text {'mum }}\right)^{2}}
$$

where $p_{n}^{\prime a n a}$ and $p_{n}^{\prime n u m}$ are the analytical and numerical pressure perturbations at point $n, N$ is the number of grids. The errors at $t=30$ are presented in Fig. 3, results show that the convergence rate of the simulations are about 4.0, which is the order of DRP scheme.

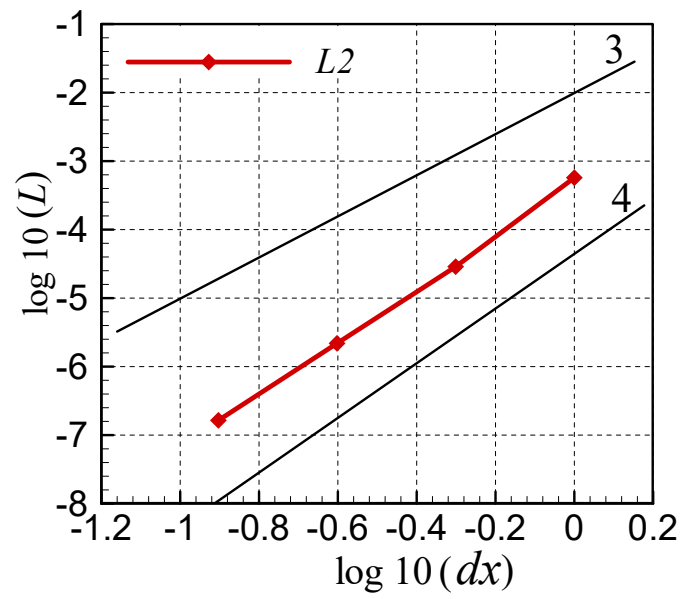

Fig. 3 Convergence curve of pressure perturbation.

\section{B. Acoustic wave scattering by a circular cylinder}

The second example is a two-dimensional acoustic wave scattered by a rigid circular cylinder [26, 37]. This case is used to test the effectiveness of immersed boundary method on solid boundaries by comparing with analytical solutions. A circular cylinder with diameter equal to 1 (reference length) is put in the center of domain, and the acoustic source is defined by a time harmonic Gaussian monopole source:

$$
S_{c o n}=\exp \left[-\ln (2) \frac{(x-4)^{2}+y^{2}}{0.2^{2}}\right] \sin (8 \pi t)
$$


The simulation is carried out with a uniform mesh $\Delta x=\Delta y=0.01$ and time step is 0.25 . The total mesh is $1200 \times 1200$, and the computational domain is $-6 \leq(x, y) \leq 6$. The pressure perturbation contour at the time of $t=42$ is presented in Fig. 4(a) and the directivities, which are the root mean square (RMS) of acoustic pressure in the time-periodic state, at $r=0.55$ and $r=5$ are plotted along with the analytical solution [37] in Fig. 4(b) and 4(c). The figure clearly demonstrates the interference pattern between emitting acoustic wave and scattering wave from the cylinder. Besides, the directivities at two positions both are corresponding well with analytical solutions, indicating the treatment on solid boundaries is accurately demonstrated.

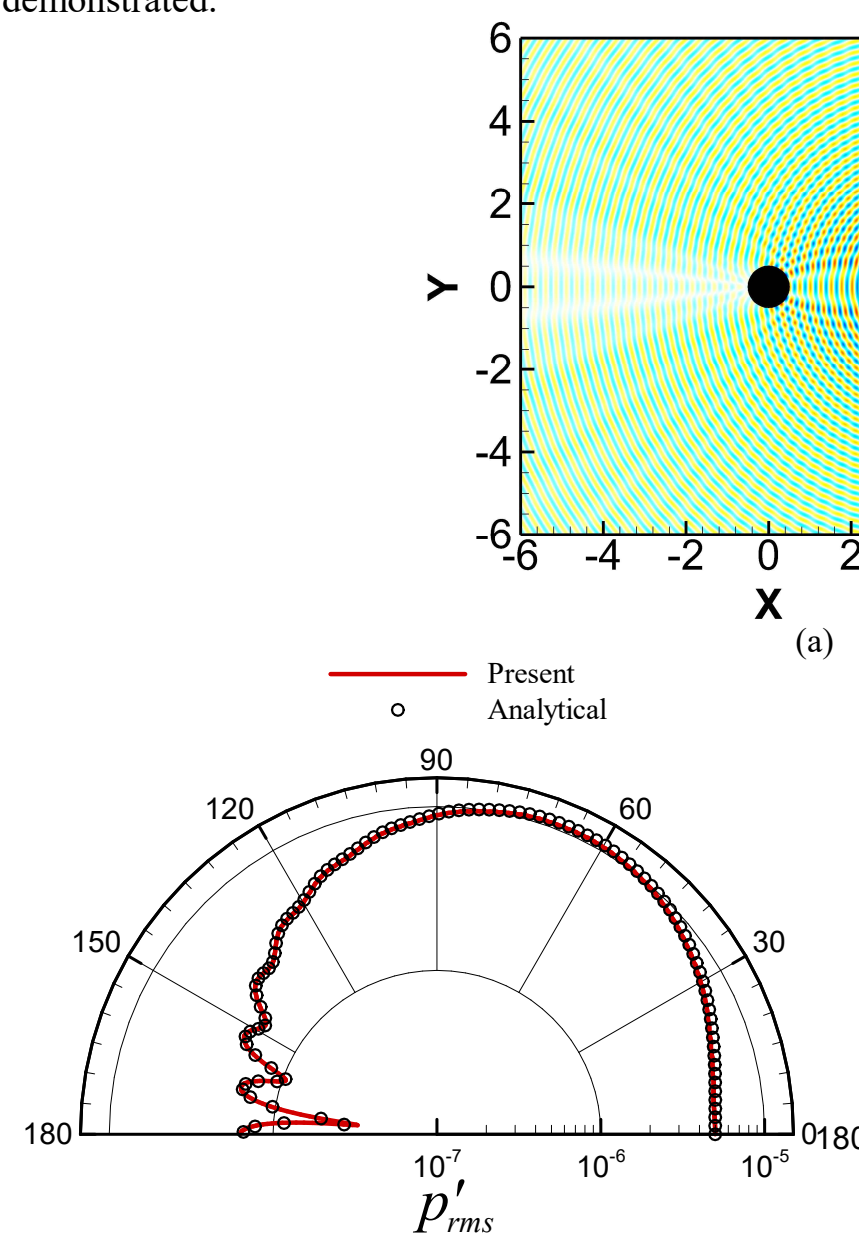

(b)

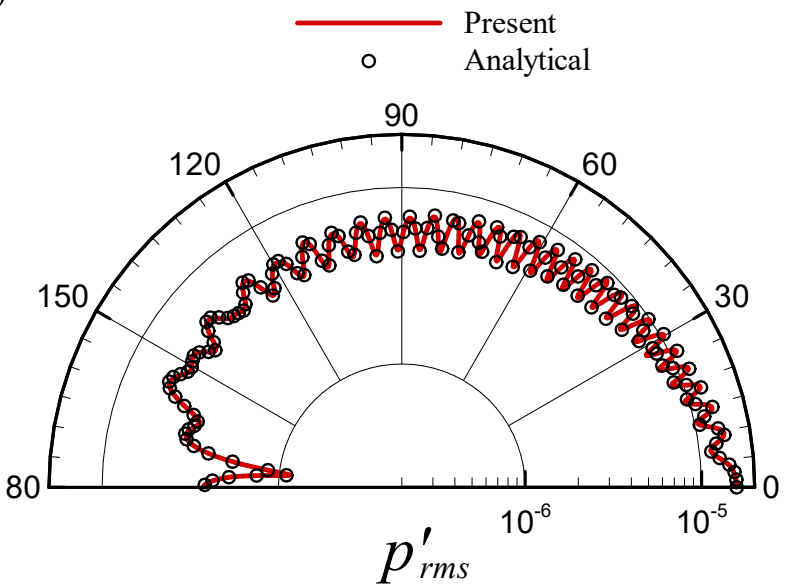

(c)

Fig. 4 Pressure perturbation contour (a) at $t=40$. Directivity of analytical and present solutions at $r$ $=0.55$ (b) and at $r=5$ (c).

\section{Acoustic wave scattering by a sphere}

To test the numerical method on dealing with three-dimensional problems, the acoustic wave scattering by a stationary sphere $[26,38,39]$ is simulated in this part. A sphere with diameter $D=1$ is placed at the center of coordinate $(0,0,0)$, and a periodic monopole Gaussian source is given by:

$$
S_{\text {cont }}=\exp \left[-(\ln 2) \frac{(x-4)^{2}+y^{2}+z^{2}}{0.2^{2}}\right] \cdot \sin (6 \pi t)
$$

In order to minimize the computational mesh, a set of non-uniform Cartesian grids are applied in this case. A uniform grid is still applied in the center of domain to capture the solid boundary, and stretching 
mesh is used away from solid. However, it should be mentioned that the maximum mesh grid cannot larger than the acoustic resolution limit, which means there must be at least 6-8 mesh per wavelength for DRP scheme. For this case, the minimum uniform grid size near the boundary is set as 0.012 and the stretching ratio is set as 1.02. In order to keep the accuracy of DRP scheme, uniform grid is used when grid size increases to 0.04 , which is about 8.3 points per wave length (PPW) for this case. The total grids are $431 \times$ $281 \times 281$ and the computation domain is about $-6.4 \leq x \leq 6.4,-3.2 \leq(y, z) \leq 3.2$. The time step is set as 0.006 . The snapshot of pressure perturbation field at $t=24$ and the directivity at $r=2$ on $z=0$ section are shown in Fig. 5(a) and 5(b), good agreements are presented with the analytical solution [39], indicating that the present methods have quite good performance for three-dimensional case. Besides, results also show that the non-uniform grid is effectiveness in the code, which has great potential to deal with complex geometry problems.

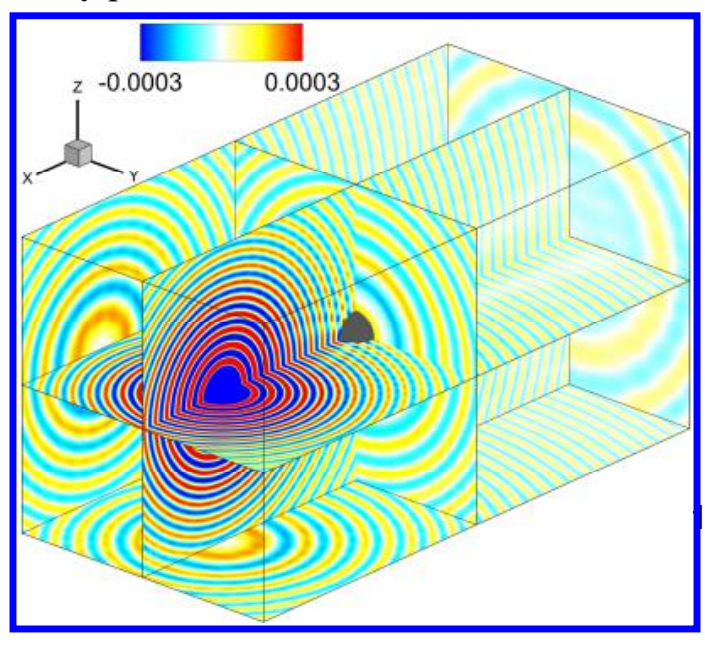

(a)

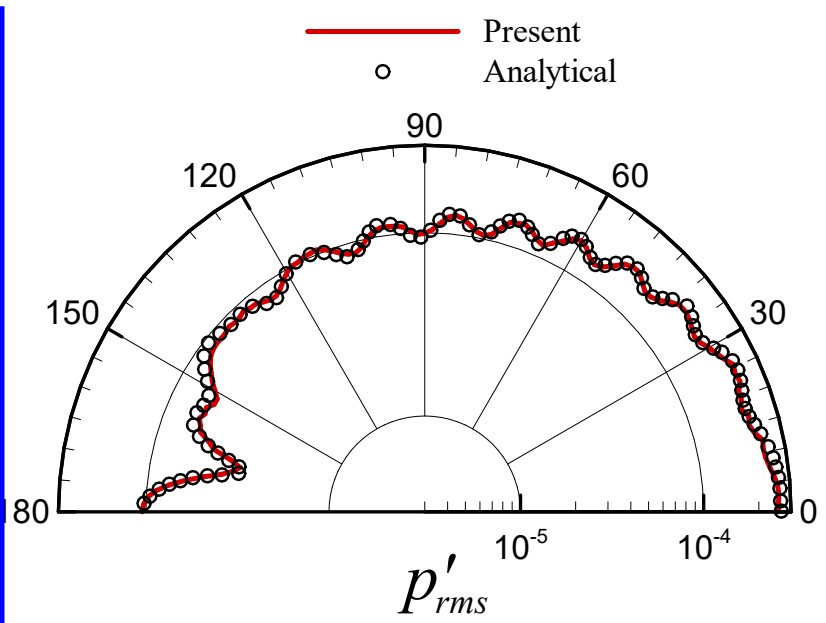

(b)

Fig. 5 (a) Pressure perturbation contour at $t=24$. (b) Directivity at $r=2$ on $z=0$ section.

\section{Flow-induces noise of flow past a circular cylinder}

All the acoustic sources of previous cases are artificial sources and the flow field is uniform for the first case. In this part, a real flow-induced noise problem is presented. The flow field is first simulated by incompressible DNS solver, with the sharp interface immersed boundary method [28], and the time-average flow and acoustic sources at every iteration are input into APEs. Both reference lengths for flow and acoustic simulation are cylinder diameter $D$. The Reynolds number based on cylinder diameter is 200 and the Mach number is 0.2 [26]. For the presented case, the same Cartesian grid is applied for flow and acoustic field. However, two grids can be different considering different requirements between flow field and acoustic simulation, and the mesh interpolation is necessary under this condition. The minimum uniform grid with $d x=d y=0.015 D$ is used around the cylinder and stretching grid with 1.03 ratio is applied outside the uniform domain, the computational grids are shown in Figure 6 . The total grids are $512 \times 512$ and the computation domain is about $0 \leq(\mathrm{x}, \mathrm{y}) \leq 104 D$. The time step of flow simulation is $0.005 \mathrm{D} / U_{\infty}$, as the nondimensional time for flow field and acoustic field is different, the time step for acoustic simulation is $0.005 \mathrm{D} / \mathrm{C}_{\infty}$, which is one fifth of flow time step. The acoustic sources at each acoustic iteration is computed by linear interpolation between two adjacent flow time steps. 


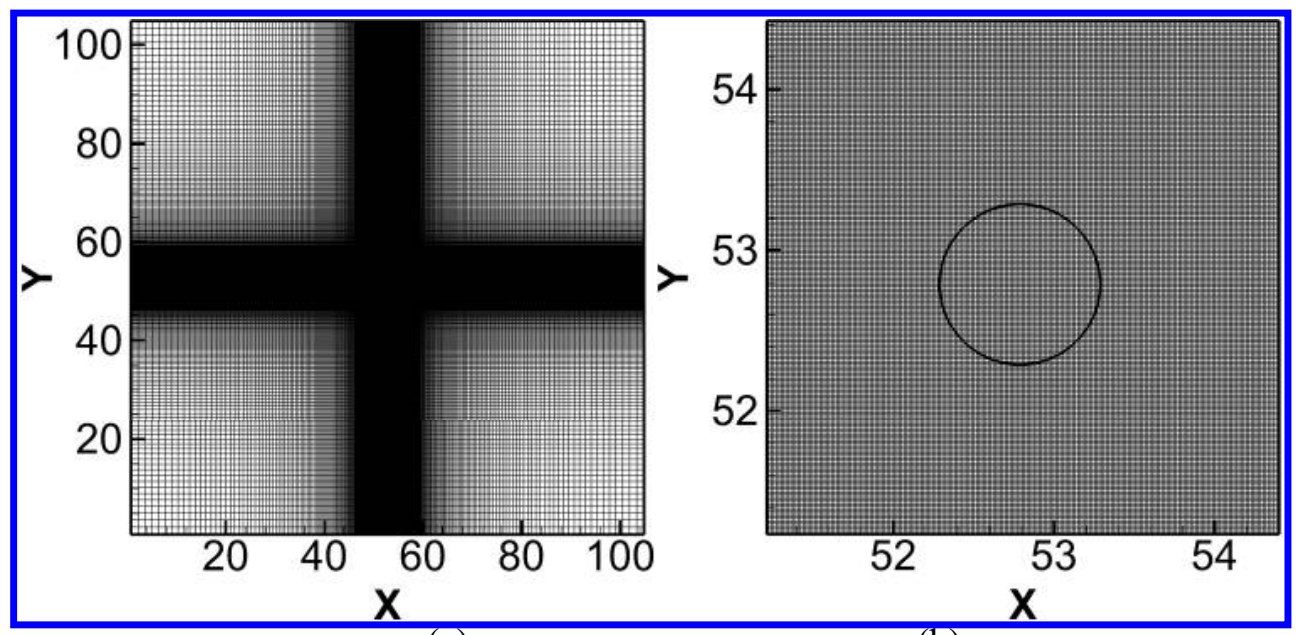

(a)

(b)

Fig. 6 (a) Computational grids of flow and acoustic simulation. (b) Densified grids around the cylinder.

The drag coefficient and lift coefficient time history are presented in Fig. 7, which are match well with reference solutions [26]. The vorticity field computed by incompressible flow solver behind the cylinder is presented in Fig. 8(a), where a clear von Kármán vortex street is observed in the cylinder wake. Besides, Fig. 8(b) shows the instantaneous acoustic pressure field, a dipole source caused by the periodic vortex shedding can be seen in the figure. The wavelength is about $25 D$, which is the expected wavelength based on vortex shedding frequency.

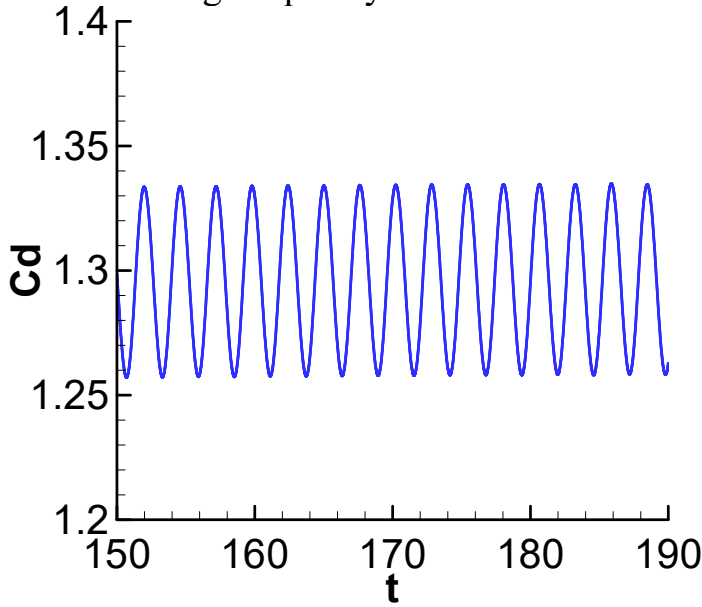

(a)

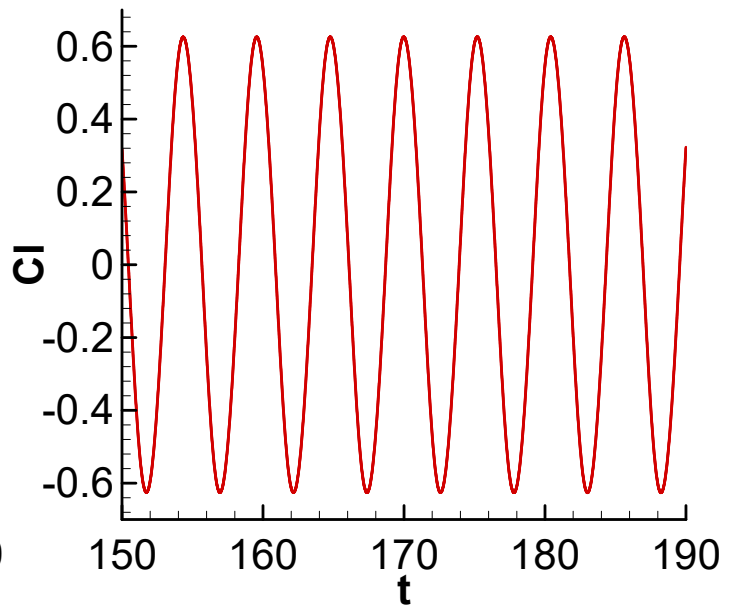

(b)

Fig. 7 Time histories of drag (a) and lift (b) coefficients. 


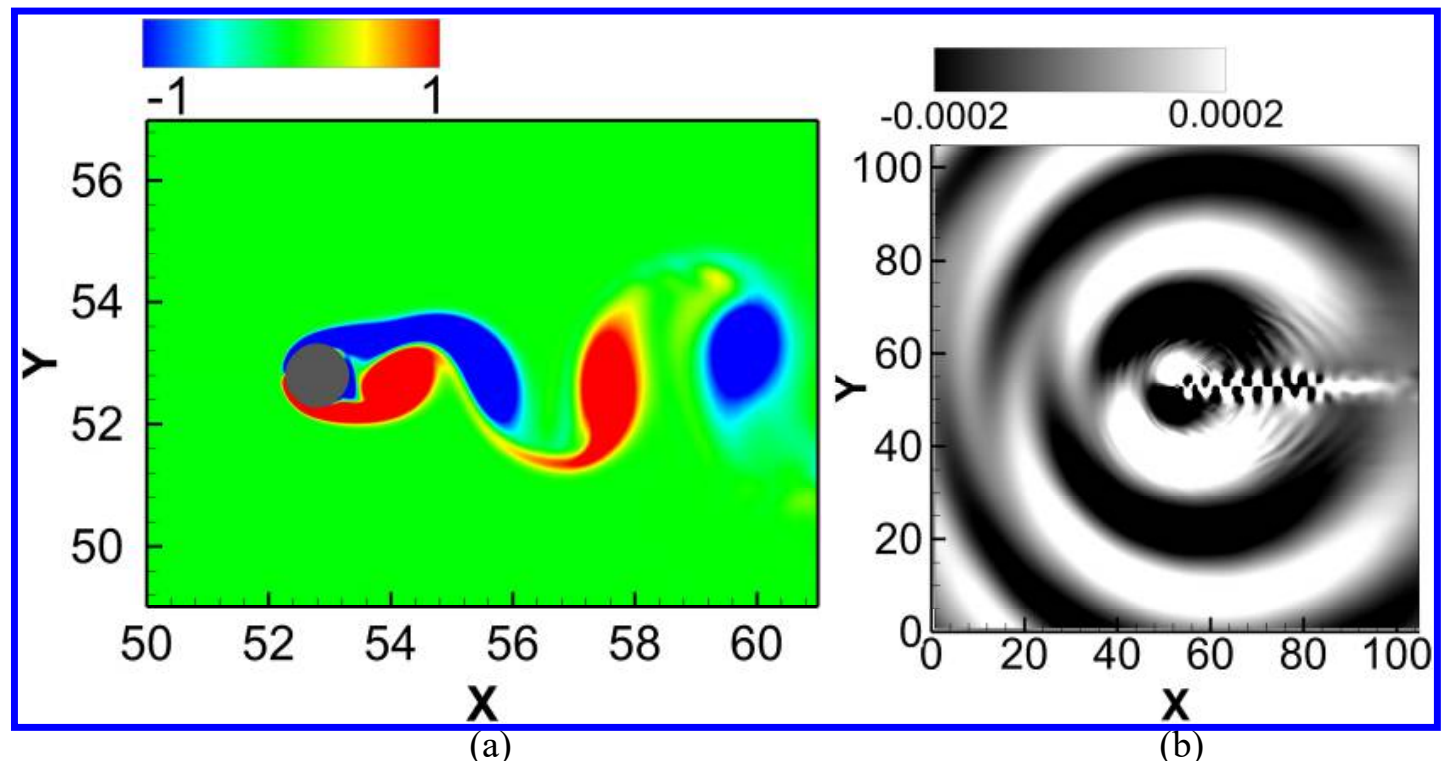

Fig. 8 (a) The vorticity field of the flow. (b) Instantaneous acoustic field.

\section{Summary}

In this paper, the sharp interface immersed boundary method is implemented to deal with acoustic scattering and flow-induced noise problems, within a hybrid computational aeroacoustic approach. An incompressible direct numerical simulation solver is first used to compute the flow field. The APEs are then solved based on sources from flow field to obtain the acoustic field. Both solid boundaries are treated by a sharp interface IBM based on a ghostpoint method. The acoustic code and numerical scheme have been validated by a Gaussian acoustic propagation problem. Besides, two benchmark acoustic scattering problems was used to validate the effectiveness IBM on twoand three-dimensional problems. Finally, a realized flow-induced noise of flow past a cylinder was computed, the wake vortex and acoustic field were simulated to validate the numerical method. More cases with moving boundaries are being worked on, which show essential potentials of the present methods.

\section{Acknowledgments}

The authors appreciate Dr. Nitin S. Dhamankar providing the codes to generate the analytical solutions of cylinder scattering problem. CZ and YY are supported by the China Scholarship Council (Nos. 201806160093, 201804910163). This study is partially supported by ONR MURI grant N00014-14-1-0533, NSF grant CBET-1605232 and NSFC (11602277).

\section{References}

[1] Triantafyllou, M. S., Triantafyllou, G., and Yue, D. "Hydrodynamics of fishlike swimming," J Annual review of fluid mechanics, Vol. 32, No. 1, 2000, pp. 33-53.

[2] Wu, T. Y. "Fish swimming and bird/insect flight," J Annual Review of Fluid Mechanics, Vol. 43, 2011, pp. 25-58.

[3] Dong, H., Bozkurttas, M., Mittal, R., Madden, P., and Lauder, G. V. "Computational modelling and analysis of the hydrodynamics of a highly deformable fish pectoral fin," Journal of Fluid Mechanics, Vol. 645, 2010, pp. 345-373.

[4] Liu, G., Ren, Y., Dong, H., Akanyeti, O., Liao, J. C., and Lauder, G. V. "Computational analysis of vortex dynamics and performance enhancement due to body-fin and fin-fin interactions in fish-like locomotion," Journal of Fluid Mechanics, Vol. 829, 2017, pp. 65-88.

[5] Wang, J., Ren, Y., Li, C., and Dong, H. "Computational investigation of wing-body interaction and its lift enhancement effect in hummingbird forward flight," Bioinspiration \& Biomimetics, Vol. 14, No. 4, 2019, p. 046010. 
[6] Li, C., and Dong, H. "Wing kinematics measurement and aerodynamics of a dragonfly in turning flight," Bioinspiration \& Biomimetics, Vol. 12, No. 2, 2017, p. 026001.

[7] Amiet, R. K. "Noise due to turbulent flow past a trailing edge," Journal of Sound and Vibration, Vol. 47, No. 3, 1976, pp. 387393.

- [8] Dowell, E. H., Gorman, G. F., and Smith, D. A. "Acoustoelasticity: General theory, acoustic natural modes and forced response to sinusoidal excitation, including comparisons with experiment," Journal of Sound and Vibration, Vol. 52, No. 4, 1977, pp. 519542 .

[9] Sueur, J., Tuck, E. J., and Robert, D. "Sound radiation around a flying fly," The Journal of the Acoustical Society of America Vol. 118, No. 1, 2005, pp. 530-8.

[10] Karamcheti, K. "Sound radiation from surface cutouts in high speed flow." California Institute of Technology, 1956.

[11] Bennet-Clark, H., and Ewing, A. "The wing mechanism involved in the courtship of Drosophila," Journal of experimental biology, Vol. 49, No. 1, 1968, pp. 117-128.

[12] Hardin, J. C., and Pope, D. S. "An Acoustic Viscous Splitting Technique for Computational Aeroacoustics," Theoretical and Computational Fluid Dynamics, Vol. 6, No. 5-6, 1994, pp. 323-340.

[13] Ewert, R., and Schroder, W. "Acoustic perturbation equations based on flow decomposition via source filtering," Journal of Computational Physics, Vol. 188, No. 2, 2003, pp. 365-398.

[14] Rowley, C. W., Colonius, T., and Basu, A. J. "On self-sustained oscillations in two-dimensional compressible flow over rectangular cavities," Journal of Fluid Mechanics, Vol. 455, 2002, pp. 315-346.

[15] Seo, J. H., and Moon, Y. J. "Linearized perturbed compressible equations for low Mach number aeroacoustics," Journal of Computational Physics, Vol. 218, No. 2, 2006, pp. 702-719.

[16] Bailly, C., and Juve, D. "Numerical solution of acoustic propagation problems using linearized Euler equations," Aiaa Journal, Vol. 38, No. 1, 2000, pp. 22-29.

-[17] Wei, M. J., and Freund, J. B. "A noise-controlled free shear flow," Journal of Fluid Mechanics, Vol. 546, 2006, pp. 123-152.

[18] Lighthill, M. J. "On sound generated aerodynamically I. General theory," Proceedings of the Royal Society of London. Series A. Mathematical and Physical Sciences, Vol. 211, No. 1107, 1952, pp. 564-587.

[19] Ffowcs Williams, J. E., and Hawkings, D. L. "Sound generation by turbulence and surfaces in arbitrary motion," Philosophical Transactions of the Royal Society of London. Series A, Mathematical Physical Sciences, Vol. 264, No. 1151, 1969, pp. 321-342.

[20] Curle, N. "The Influence of Solid Boundaries Upon Aerodynamic Sound," Proceedings of the Royal Society of London. Series A. Mathematical and Physical Sciences, Vol. 231, No. 1187, 1955, pp. 505-514.

-[21] Wang, M., Freund, J. B., and Lele, S. K. "Computational prediction of flow-generated sound," Annual Review of Fluid Mechanics, Vol. 38, 2006, pp. 483-512.

[22] Bogey, C., Bailly, C., and Juvé, D. J. A. j. "Computation of flow noise using source terms in linearized Euler's equations," Aiaa Journal, Vol. 40, No. 2, 2002, pp. 235-243.

[23] Shen, W. Z., and Sorensen, J. N. "Comment on the aeroacoustic formulation of Hardin and Pope," Aiaa Journal, Vol. 37, No. 1, 1999, pp. 141-143.

- [24] Shen, W. Z., and Sorensen, J. N. "Aeroacoustic modelling of low-speed flows," Theoretical and Computational Fluid Dynamics, Vol. 13, No. 4, 1999, pp. 271-289.

[25] Ewert, R., and Schroder, W. "On the simulation of trailing edge noise with a hybrid LES/APE method," Journal of Sound and Vibration, Vol. 270, No. 3, 2004, pp. 509-524.

[26] Seo, J. H., and Mittal, R. "A high-order immersed boundary method for acoustic wave scattering and low-Mach number flowinduced sound in complex geometries," Journal of Computational Physics, Vol. 230, No. 4, 2011, pp. 1000-1019. 
[27] Geng, B., Xue, Q., Zheng, X., Liu, G., Ren, Y., and Dong, H. "The effect of wing flexibility on sound generation of flapping wings," Bioinspiration \& Biomimetics, Vol. 13, No. 1, 2017, p. 016010.

- [28] Mittal, R., Dong, H., Bozkurttas, M., Najjar, F. M., Vargas, A., and von Loebbecke, A. "A versatile sharp interface immersed boundary method for incompressible flows with complex boundaries," Journal of Computational Physics, Vol. 227, No. 10, 2008, pp. $4825-4852$.

[29] Wang, J., Xi, J., Han, P., Wongwiset, N., Pontius, J., and Dong, H. "Computational analysis of a flapping uvula on aerodynamics and pharyngeal wall collapsibility in sleep apnea," Journal of biomechanics, Vol. 94, 2019, pp. 88-98.

-[30] Tam, C. K. W., and Webb, J. C. "Dispersion-Relation-Preserving Finite Difference Schemes for Computational Acoustics," Journal of Computational Physics, Vol. 107, No. 2, 1993, pp. 262-281.

[31] Tam, C. K. W. "Computational aeroacoustics - Issues and methods," Aiaa Journal, Vol. 33, No. 10, 1995, pp. 1788-1796.

[32] Bogey, C., and Bailly, C. "A family of low dispersive and low dissipative explicit schemes for flow and noise computations," Journal of Computational Physics, Vol. 194, No. 1, 2004, pp. 194-214.

[33] Hu, F. Q., Hussaini, M. Y., and Manthey, J. L. "Low-Dissipation and Low-Dispersion Runge-Kutta Schemes for Computational Acoustics," Journal of Computational Physics, Vol. 124, No. 1, 1996, pp. 177-191.

[34] Tam, C. K. W., and Dong, Z. "Radiation and outflow boundary conditions for direct computation of acoustic and flow disturbances in a nonuniform mean flow," Journal of Computational Acoustics, Vol. 4, No. 2, 1996, pp. 175-201.

[35] Bogey, C., and Bailly, C. "Three-dimensional non-reflective boundary conditions for acoustic simulations: far field formulation and validation test cases," Acta Acustica United with Acustica, Vol. 88, No. 4, 2002, pp. 463-471.

[36] Hardin, J., Ristorcelli, J., and Tam, C. "ICASE/LaRC workshop on benchmark problems in computational aeroacoustics," National Aeronautics Space Administration, 1994.

[37] Tam, C. K., and Hardin, J. C. "Second computational aeroacoustics (CAA) workshop on benchmark problems," 1997.

[38] Fukushima, Y., Sasaki, D., and Nakahashi, K. "Cartesian Mesh Linearized Euler Equations Solver for Aeroacoustic Problems around Full Aircraft," International Journal of Aerospace Engineering, Vol. 2015, 2015.

[39] Morris, P. J. "Scattering of sound from a spatially distributed, spherically symmetric source by a sphere," The Journal of the Acoustical Society of America, Vol. 98, No. 6, 1995, pp. 3536-3539. 